\title{
Treatment-induced neuropathy of diabetes related to abrupt glycemic control
}

\author{
Meggie Stainforth-Dubois MD, Emily G. McDonald MD MSc
}

Cite as: CMAJ 2021 July 19;193:E1085-88. doi: 10.1503/cmaj.202091

A 20-year-old woman with a history of generalized anxiety disorder presented to the emergency department with several months' history of polyuria and polydipsia and a 10-kg weight loss (current weight: $47.8 \mathrm{~kg}$ ). The patient had no history of substance use or disordered eating, and her family history was unremarkable. Her blood glucose level was 40 (normal range 3.9-11) $\mathrm{mmol} / \mathrm{L}$ and her hemoglobin $\mathrm{A}_{1 \mathrm{c}}\left(\mathrm{HbA}_{1 \mathrm{c}}\right)$ level was $17 \%$ [normal range $4.3 \%-6 \%$ ]. She was admitted to hospital with a diagnosis of new-onset diabetes mellitus (presumed type 1) and treatment with basal and prandial insulin was started (i.e., insulin degludec, 6 units at bedtime; insulin lispro, 3 units before breakfast, lunch and dinner), with no correction doses. The patient's level of anti-glutamic acid decarboxylase (GAD) antibodies, which lead to pancreatic $\beta$-cell destruction, was elevated (5.7 [normal range $0-4.9] \mathrm{U} / \mathrm{mL}$ ), which confirmed autoimmune diabetes. Her symptoms and blood glucose improved over 48 hours, and she was discharged home.

Over the course of 1 month the endocrinology team titrated the patient's insulin progressively to optimize glycemic control to a dose of insulin degludec 22 units before bedtime, and insulin lispro 9 units before breakfast and 10 units before lunch and dinner (Figure 1). The nutrition team provided further dietary counselling. After several weeks of treatment, the patient had recurrent episodes of mild symptomatic hypoglycemia, diagnosed with home glucose monitoring. As a consequence, her insulin required several dose reductions. Three months into treatment the symptoms had resolved for the most part; the patient was taking insulin degludec 18 units before bedtime, and insulin lispro 2 units before breakfast and dinner and 5 units before lunchtime.

Several diabetes- and treatment-associated complications developed in our patient. After 3 months of treatment, the patient's $\mathrm{HbA}_{1 \mathrm{c}}$ level was $6.7 \%$. Around this time, she reported episodes of severe dizziness and we diagnosed orthostatic hypotension (supine blood pressure of $115 / 83 \mathrm{~mm} \mathrm{Hg}$ compared with standing blood pressure of $76 / 59 \mathrm{~mm} \mathrm{Hg}$ ). The main differential diagnoses were adrenal insufficiency and treatment-induced neuropathy of diabetes. The endocrinology team ordered tests for early morning cortisol, $\mathrm{B}_{12}$, thyroid-stimulating hormone and antineuronal antibodies, and treated her symptoms with midodrine (5 mg 3 times daily) and fludrocortisone (0.05 mg twice a day).

\section{KEY POINTS}

- Rapid correction of glycemic control (i.e., > 2 percentage points of hemoglobin $A_{1 c}$ ) over 3 months may lead to treatment-induced neuropathy of diabetes.

- Symptoms include painful neuropathy, autonomic dysfunction, gastroparesis, early worsening of retinopathy and microalbuminuria.

- Women and people with type 1 diabetes are at elevated risk of treatment-induced neuropathy of diabetes.

- Management of the condition may include involvement of a dietitian, adjustment of insulin dose and use of medications to improve symptoms such as painful neuropathy, postural hypotension and gastroparesis.

They also referred her to the neurology service for assessment of possible treatment-induced neuropathy of diabetes.

All laboratory test results at this stage were normal. Autonomic reflex testing showed reduced heart oscillations at rest, and an attenuated cardiomotor response to deep breathing and the Valsalva maneuvre, consistent with autonomic dysfunction. A head-up tilt test showed substantial orthostatic hypotension. The neurology team confirmed a diagnosis of treatment-induced neuropathy of diabetes. Shortly after this, the patient developed lancinating pain in her lower extremities. Nerve conduction studies confirmed large and small fibre sensory neuropathy. The endocrinology team prescribed venlafaxine, with the aim of relieving both her pain and anxiety. The neurology team also suggested retinal screening, and mild early proliferative retinopathy that did not require immediate intervention was diagnosed.

Six months after diagnosis, our patient developed intractable nausea and vomiting. Abdominal ultrasonography, gastroscopy and abdominal computed tomography were unremarkable. Despite treatment with metoclopramide, her symptoms persisted, necessitating repeated hospital admissions. A gastric emptying study confirmed delayed liquid emptying and a diagnosis of gastroparesis was made (Figure 2). Working with a clinical nutritionist, the patient tried a gastroparesis diet, but found it difficult to adhere to. Various medications including ondansetron, domperidone, erythromycin, nabilone, ketamine, prucalopride and 
Clinical history

$\begin{array}{ccc}\text { Diagnosis } & \text { Orthostatic } & \text { Nausea/ } \\ \text { of diabetes } & \text { symptoms } & \text { vomiting }\end{array}$

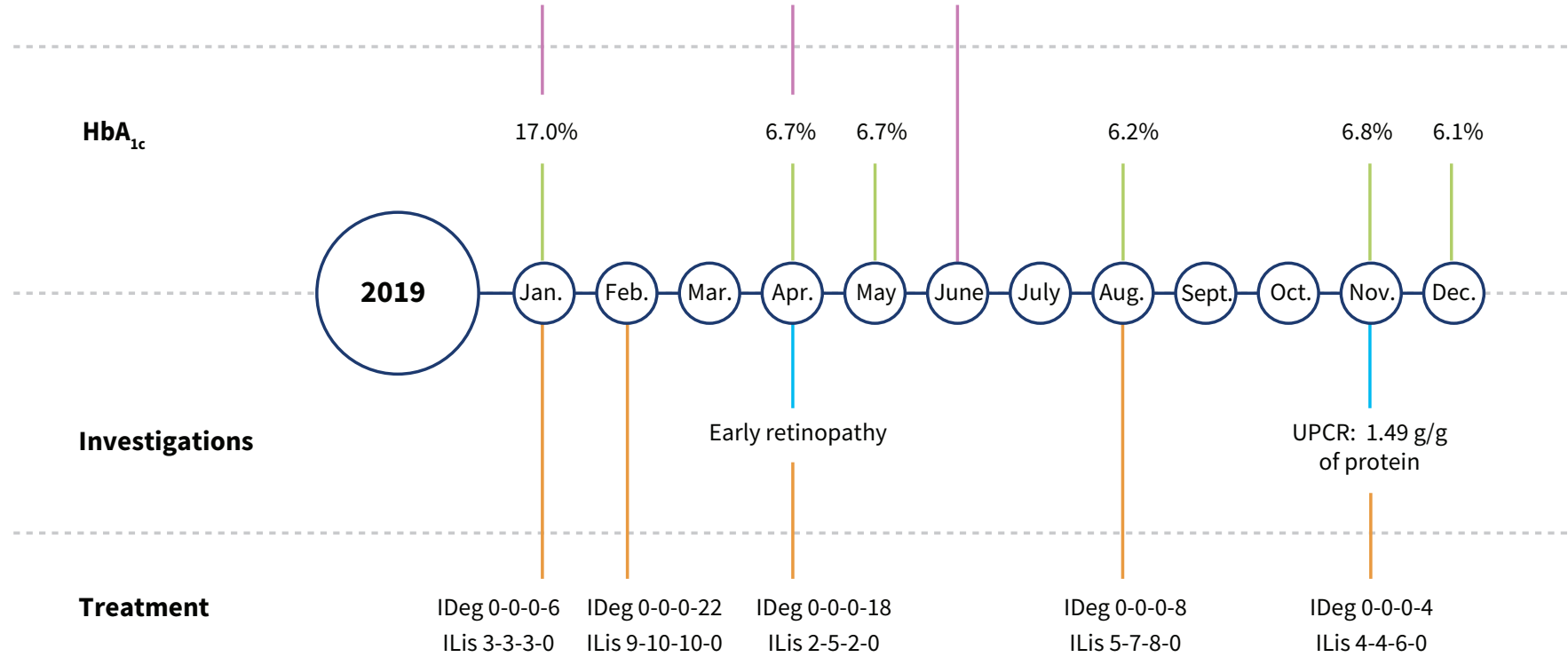

Figure 1: Timeline illustrating the order of events including clinical history, hemoglobin $A_{1 c}\left(\mathrm{HbA}_{1 c}\right)$, investigations and treatment regimens. Insulin dosing is represented as follows: 0-0-0-6 refers to 6 units at bedtime, and 3-3-3-0 refers to 3 units before breakfast, lunch and dinner. Note: IDeg = insulin degludec, ILis = insulin lispro, UPCR = urine protein-to-creatinine ratio.

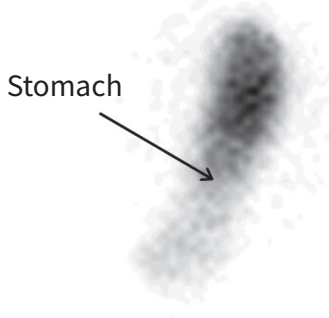

$1 \mathrm{~min}$

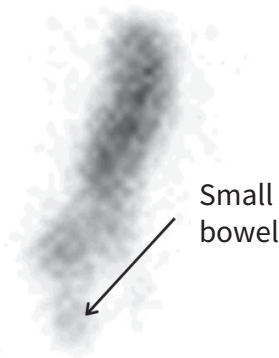

$10 \mathrm{~min}$

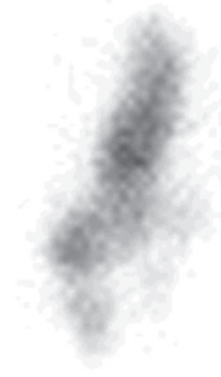

$20 \min$

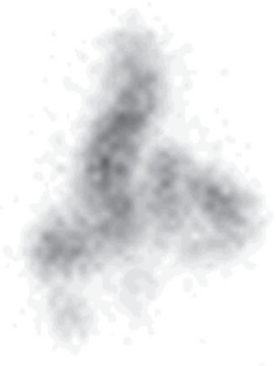

$30 \mathrm{~min}$

Figure 2: Liquid gastric emptying study showing a substantially reduced emptying rate. Normally, $50 \%$ of the stomach contents will have emptied after 6-20 minutes. Half-time is extrapolated to be 57 minutes.

haloperidol were unsuccessful in relieving her symptoms. Intrapyloric injections of botulinum toxin had little effect. Ten months after treatment began, microalbuminuria developed (1.49 [normal range $<0.15] \mathrm{g} / \mathrm{g}$ ), although her glomerular filtration rate as unaffected.

Over the next year and a half, the patient's orthostatic hypotension improved and her endocrinologist stopped midodrine. She reported adequate pain management with venlafaxine, but her symptoms of nausea and vomiting persisted. She remains on regular domperidone and ondansetron, with haloperidol as needed. Her retinopathy has improved, her microalbuminuria returned to a normal level and her $\mathrm{HbA}_{\mathrm{cc}}$ has ranged between $6.1 \%$ and $7.1 \%$.

\section{Discussion}

Treatment-induced neuropathy of diabetes is defined by the onset of neuropathy after aggressive glycemic control. Previously known as "insulin neuritis," it has been linked to any pharmacologic or lifestyle intervention (including rapid weight loss and intense caloric restriction) that leads to an overly rapid correction of $\mathrm{HbA}_{1 c} .{ }^{1}$ According to a 2015 retrospective review involving 954 patients who were followed for diabetic neuropathy in Boston, as many as $10 \%$ of patients with diabetes had an abrupt increase in neuropathic pain or autonomic symptoms after a rapid change in glucose control, which suggests that this diagnosis has been underreported. ${ }^{2}$ The study defined rapid 
change as greater than $2 \mathrm{HbA}_{1 \mathrm{c}}$ percentage points over 3 months. Among patients with diabetes whose $\mathrm{HbA}_{1 \mathrm{c}}$ had been lowered at a rate of less than 2 percentage points over 3 months, the risk of neuropathic symptoms was $4.3 \%$. Several other studies, including one that described the syndrome in women with eating disorders, corroborated an increased risk of treatment-induced neuropathy of diabetes after rapid correction of $\mathrm{HbA}_{1 \mathrm{c}}{ }^{1,3-6}$

The mechanisms leading to treatment-induced neuropathy of diabetes are thought to be related to apoptosis induced by sudden glucose deprivation, the formation of arteriovenous shunts causing endoneural ischemia and regeneration of nerve fibres causing pain..$^{4,7}$ It occurs more frequently in patients with type 1 than in those with type 2 diabetes: 1 study reported that 76 out of 104 patients with treatment-induced neuropathy of diabetes had type $1 .^{2}$ Susceptible patients often have a long-standing history of hyperglycemia that, on average, dates back 5-10 years. ${ }^{2}$

The principal clinical manifestation of treatment-induced neuropathy of diabetes is neuropathic pain. ${ }^{1}$ Orthostatic hypotension is reported in $69 \%$ of cases, more frequently with type 1 than with type 2 diabetes. ${ }^{5}$ Gastrointestinal symptoms are common; ${ }^{3} 1$ small study involving 16 patients who presented with acute painful neuropathy reported nausea in $69 \%$ of patients and vomiting in 56\%. ${ }^{5}$ However, it is somewhat atypical for the gastrointestinal symptoms to overshadow the pain from peripheral neuropathy, as in our patient.

Data on the effects of aggressive glycemic control on microvascular complications are limited. In the Diabetes Control and Complications Trial, which involved 1441 patients with type 1 diabetes, early retinopathy developed in $22 \%$ of patients with intensive insulin therapy compared with $13 \%$ in the conventional therapy group. ${ }^{4,8}$ This occurred as early as 3 months after the start of treatment. ${ }^{8}$ Despite this observation, intensive glycemic control led to overall improved results over 18 years. ${ }^{8}$ In 1 retrospective study involving 104 patients with treatment-induced neuropathy of diabetes, $17 \%$ had microalbuminuria at baseline compared with $84 \%$ at 1 year after the diagnosis of neuropathy. ${ }^{2}$ Elevations in creatinine level are uncommon. ${ }^{1}$

\section{Management and prognosis}

Data are lacking to guide the optimal management of treatmentinduced neuropathy of diabetes. Current expert opinion, based only on observational data, is to avoid this neuropathy by limiting the rate of reduction in $\mathrm{HbA}_{1 \mathrm{c}}$ to less than 2 percentage points (e.g., from 8 to 6 ) over 3 months. ${ }^{2}$ In practice, however, this can be difficult to achieve and is debatable. Some case reports and series have shown that painful neuropathy may improve substantially after loosening of glycemic control; 1 study also suggested improvement in early diabetic retinopathy. ${ }^{1,9}$ Therefore, some experts suggest a period of permissive hyperglycemia as a "salvage treatment," whereas others have questioned this approach. ${ }^{6,10}$ A small longitudinal cohort study followed 26 patients with treatment-induced neuropathy of diabetes for 8 years. ${ }^{1}$ All 19 patients who maintained stable, adequate glycemic control $\left(\mathrm{HbA}_{1 \mathrm{c}}\right.$ between $6 \%$ and $\left.8.5 \%\right)$ after the development of symptoms of treatment-induced neuropathy of diabetes had gradual improvement in their neuropathic symptoms over several years, with near-complete functional recovery, which suggests that the syndrome is reversible to some degree. Conversely, all 7 patients with poor, unstable glycemic control had worsening neuropathy, retinopathy and nephropathy.

Despite increasing awareness of treatment-induced neuropathy of diabetes, there are not enough data to suggest a uniform approach to treatment at this time. The current standard of care remains supportive and includes avoidance of overly rapid correction when starting treatment, especially in patients with risk factors such as type 1 diabetes or a long-standing history of hyperglycemia; women and people with eating disorders are also at risk.

Supportive care currently resembles traditional management of similar complications in patients with long-standing poor glycemic control. Appropriate workup of painful neuropathy and autonomic dysfunction may include referral to a neurologist for specialized testing. Treatment typically involves the use of a single neuropathic agent (i.e., an antiepileptic or antidepressant) but may sometimes require combination therapy. ${ }^{4}$ Tricyclic antidepressants, if administered, must be used with caution because they may exacerbate orthostatic hypotension. ${ }^{4}$

Regarding symptoms related to gastroparesis, all patients with suspected delayed gastric emptying should be referred to gastroenterology for endoscopy and consideration for abdominal imaging to exclude gastric outlet obstruction. ${ }^{11}$ Formal diagnosis requires a scintigraphic gastric emptying study, ideally with solids if tolerated. ${ }^{11}$ Involvement of a nutritionist is recommended. Firstline treatment usually involves dietary modifications (low-fat, low-fibre, small-particle diet) and promotility medications such as metoclopramide or domperidone that must be monitored because of their potential adverse effects. ${ }^{11}$ Antiemetics such as ondansetron or diphenhydramine are often added to treatment regimens in patients with refractory symptoms. ${ }^{11}$ Erythromycin, a motilin agonist, can also be used, although its long-term efficacy is limited by tachyphylaxis. ${ }^{11}$ Serotonin receptor antagonists, such as prucalopride, if tolerated, can improve rates of gastric emptying. ${ }^{11}$ Nabilone and haloperidol were used for our patient because of their ability to control chemotherapy-induced nausea and vomiting, although they are not typically indicated in gastroparesis. Refractory symptoms may warrant consideration of more invasive therapies such as gastric electrical stimulation. ${ }^{11}$

\section{References}

1. Gibbons $\mathrm{CH}$. Treatment induced neuropathy of diabetes - long term implications in type 1 diabetes. J Diabetes Complications 2017;31:715-20.

2. Gibbons $\mathrm{CH}$, Freeman R. Treatment-induced neuropathy of diabetes: an acute, iatrogenic complication of diabetes. Brain 2015;138:43-52.

3. Alexandrou EG, Corathers SD, Lahoti A, et al. Treatment-induced neuropathy of diabetes in youth: case series of a heterogeneous and challenging complication. J Endocr Soc 2020;4:bvaa154. doi: 10.1210/jendso/bvaa154.

4. Gibbons $\mathrm{CH}$. Treatment induced neuropathy of diabetes. Auton Neurosci 2020;226:102668.

5. Gibbons $\mathrm{CH}$, Freeman R. Treatment induced diabetic neuropathy - a reversible painful autonomic neuropathy. Ann Neurol 2010;67:534-41.

6. Siddique N, Durcan R, Smyth S, et al. Acute diabetic neuropathy following improved glycaemic control: a case series and review. Endocrinol Diabetes Metab Case Rep 2020;2020:19-0140. doi: 10.1530/EDM-19-0140. 
7. Honma H, Podratz JL, Windebank A. Acute glucose deprivation leads to apoptosis in a cell model of acute diabetic neuropathy. J Peripher Nerv Syst 2003;8:65-74.

8. Bain SC, Klufas MA, Ho A, et al. Worsening of diabetic retinopathy with rapid improvement in systemic glucose control: a review. Diabetes Obes Metab 2019;21:454-66.

9. Chantelau E, Meyer-Schwickerath R. Reversion of 'early worsening' of diabetic retinopathy by deliberate restoration of poor metabolic control. Ophthalmologica 2003;217:373-7.

10. Chandler E, Brown M, Wintergerst K, et al. Treatment-induced neuropathy of diabetes in pediatrics: a case report and review of the literature. J Clin Endocrinol Metab 2020;105:395-8.

11. Bharucha AE, Kudva YC, Prichard DO. Diabetic gastroparesis. Endocr Rev 2019;40:1318-52.
The section Cases presents brief case reports that convey clear, practical lessons. Preference is given to common presentations of important rare conditions, and important unusual presentations of common problems. Articles start with a case presentation (500 words maximum), and a discussion of the underlying condition follows (1000 words maximum). Visual elements (e.g., tables of the differential diagnosis, clinical features or diagnostic approach) are encouraged. Consent from patients for publication of their story is a necessity. See information for authors at www. cmaj.ca.

\section{Competing interests: None declared.}

This article has been peer reviewed.

The authors have obtained patient consent.

Affiliations: Department of Medicine (Stainforth-Dubois, McDonald), Clinical Practice Assessment Unit (McDonald), McGill University Health Centre, Montréal, Que.
Contributors: Both authors contributed substantially to the acquisition and analysis of data, and drafting and revision of the manuscript; gave final approval of the version to be published; and agreed to be accountable for all aspects of the work.

Content licence: This is an Open Access article distributed in accordance with the terms of the Creative Commons Attribution (CC BY-NC-ND
4.0) licence, which permits use, distribution and reproduction in any medium, provided that the original publication is properly cited, the use is noncommercial (i.e., research or educational use), and no modifications or adaptations are made. See: https://creativecommons. org/licenses/by-nc-nd/4.0/

Correspondence to: Meggie Stainforth-Dubois, meggie.stainforth-dubois@mail.mcgill.ca 